\title{
On the zeros of blocked time-invariant systems
}

\author{
Mohsen Zamani ${ }^{\mathrm{a}, *}$, Brian D.O. Anderson ${ }^{\mathrm{a}, \mathrm{b}}$, Uwe Helmke ${ }^{\mathrm{c}}$, Weitian Chen ${ }^{\mathrm{d}}$ \\ a Research School of Engineering, Australian National University, Canberra, ACT 0200, Australia \\ ${ }^{\mathrm{b}}$ Canberra Research Laboratory, National ICT Australia Ltd., PO Box 8001, Canberra, ACT 2601, Australia \\ ${ }^{c}$ Department of Mathematics, University of Würzburg, 97074 Würzburg, Germany \\ ${ }^{\mathrm{d}}$ Department of Electrical and Computer Engineering, Faculty of Engineering, University of Windsor, Windsor, ON N9B 3P4, Canada
}

\section{A R T I C L E I N F O}

\section{Article history:}

Received 30 May 2012

Received in revised form

12 April 2013

Accepted 15 April 2013

Available online 17 May 2013

\section{Keywords:}

Linear systems theory

Multirate systems

Blocked systems

Zeros

\begin{abstract}
A B S T R A C T
This paper studies the zero properties of blocked linear systems resulting from blocking of linear timeinvariant systems. The main idea is to establish a relation between the zero properties of blocked systems and the zero properties of their corresponding unblocked systems. In particular, it is shown that the blocked system has a zero if and only if the associated unblocked system has a zero. Furthermore, the zero properties of blocked systems under a generic setting i.e. a setting which parameter matrices $A, B, C, D$ assume generic values, are examined. It is demonstrated that nonsquare blocked systems i.e. blocked systems with number of outputs unequal to the number of inputs, generically have no zeros; however, square blocked systems i.e. blocked systems with equal number of inputs and outputs, generically have only finite zeros and these finite zeros have geometric multiplicity one.
\end{abstract}

Crown Copyright @ 2013 Published by Elsevier B.V. All rights reserved.

\section{Introduction}

The well-known technique of blocking or lifting has been developed in systems and control [1] and signal processing [2]. In the systems and control literature, this method has mostly been exploited to transform linear discrete-time periodic systems to linear time-invariant systems so that the well-developed tools for linear time-invariant systems can be extended for design and analysis of linear discrete-time periodic systems [3-6]. For example, the authors in $[3,4]$ have extended the notions of poles and zeros of linear time-invariant systems to linear periodic systems. Some necessary and sufficient conditions for structural properties such as observability and reachability have been studied in $[7,8]$. Moreover, the realization problem has been researched in [8] and the related references listed therein.

The blocking technique has been applied to linear timeinvariant systems as well; see e.g. [1,9] and the references therein. For instance, in [9], linear time-invariant systems have been blocked for the purpose of designing periodic controllers while the authors in [1] have performed the blocking technique on linear time-invariant systems for the purpose of dealing with multirate sampled-data systems.

\footnotetext{
* Corresponding author. Tel.: +61 261258685.

E-mail addresses: mohsen.zamani@anu.edu.au, zamani.moh@gmail.com (M. Zamani), brian.anderson@anu.edu.au (B.D.O. Anderson), helmke@mathematik.uni-wuerzburg.de(U. Helmke), cwt6122@uwindsor.ca (W. Chen).
}

In this paper, we examine the zero properties of the blocked systems resulting from blocking of linear time-invariant systems. This study is motivated from both application and theoretical perspectives. As mentioned above, the blocking of linear time-invariant systems is useful in the multirate sampled-data systems controller design as shown by $[1,9]$. Furthermore, from a theoretical perspective, the pole properties of the blocked systems are well understood [6,9], whereas much less known about the zero properties of the blocked systems. Ref. [6] studies the zero properties of blocked systems obtained from blocking of linear periodic systems. The results show that the blocked system has a finite zero if the related linear time-invariant unblocked system has a finite zero, which is a form of sufficiency result. However, this reference does not provide a necessary condition for the blocked system to have a finite zero; also, zeros at infinity are not considered. These gaps have been covered in our works [10,21] where we have introduced some additional results about the zero properties of blocked systems. For instance, in [10] matrix fraction descriptions (MFDs) have been used to establish a relation between the zero properties of blocked systems and the zero properties of their corresponding unblocked systems. Moreover, in [21], the time domain approach has been exploited to explore the zero properties of blocked systems. Both $[10,21]$ only have considered tall blocked systems i.e. blocked systems with more outputs than inputs, and they show that tall blocked systems have a zero if and only if the corresponding unblocked systems have a zero. Furthermore, in [10,21] only blocked systems for which their associated transfer functions have fullcolumn normal rank, have been explored. 
In this paper, we generalize the results of $[10,21]$. The zero properties of a general blocked system are studied. Here, there exists no assumption such as tallness or fatness on the structure of blocked systems. Furthermore, we relax the assumption used in [10,21] on the normal rank of the transfer function associated with the blocked system. In both aforementioned references i.e. [10,21], the normal rank of the transfer function is assumed to be equal to its number of columns; however, in the current paper, we put no assumption on the normal rank of the transfer function associated with the blocked system. Moreover, we explore the zero properties of a general blocked system for all possible choices of zeros i.e. finite nonzero system zeros, system zeros at zero and system zeros at infinity. It is clearly shown that for all possible choices of zeros, the blocked system has a zero if and only if its corresponding unblocked system has a zero.

In addition, the zero properties of blocked systems are examined for a generic choice of matrices $A, B, C$ and $D$ in a minimal state space representation corresponding to the nonzero, nonconstant transfer function $D+C(z I-A)^{-1} B$. It is important to mention that the zero properties of unblocked linear time-invariant systems under a genericity assumption have been studied in the literature (see for e.g. [11-17]). For instance, [11] states that the existence of a nontrivial great common divisor among a set of polynomials is a nongeneric property. Furthermore, the zero properties of unblocked systems have been also examined under a genericity assumption in $[13,15]$. It is known that nonsquare unblocked systems i.e. unblocked systems with number of outputs unequal to the number of inputs, are generically zero-free $[15,12,13]$ p. 448. [15] has exploited the state space approach to show that tall unblocked systems i.e. unblocked systems with more number of outputs than inputs, are generically zero-free. However, square unblocked systems i.e. unblocked systems with equal number of inputs and outputs, generically have only finite zeros $[14,15]$. These results cannot immediately be applied to the blocked case, because parameter matrices of blocked systems are structured and their entries cannot then be independently assigned.

In this paper, we particularly show that nonsquare blocked systems i.e. blocked systems with number of outputs unequal to the number of inputs, when parameter matrices $A, B, C$ and $D$ assume generic values, have no zeros. However, when one is considering generic square blocked systems i.e. blocked systems with equal number of inputs and outputs and the matrices $A, B, C$ and $D$ take generic values, it is shown that square blocked systems have no infinite zeros but they have finite zeros and the kernel of the system matrix associated with any zero is one dimensional.

The structure of this paper is as follows. First, in Section 2 we provide the problem formulation. Then a relation between the transfer function of blocked systems and the transfer function of the associated unblocked systems is recalled. Based on the relation obtained in Section 2, Section 3 relates the zeros of blocked systems to the zeros of their corresponding unblocked systems. Section 3 also studies the zero properties of blocked systems under the genericity assumption. Finally, Section 4 provides the concluding remarks.

\section{Blocked systems and unblocked systems-the state space representation and the transfer function}

In this section, first a formulation for the problem under study is given. Then a relation between the unblocked system transfer function and the blocked system transfer function is established. The obtained relation is then used in the next section for the analysis of the blocked system zeros.

\subsection{Unblocked systems and blocked systems-the state space}

The linear time-invariant unblocked system under consideration is described as

$x_{k+1}=A x_{k}+B u_{k}$

$y_{k}=C x_{k}+D u_{k}$,

where $k \in \mathbb{Z}, x_{k} \in \mathbb{R}^{n}, y_{k} \in \mathbb{R}^{p}$ and $u_{k} \in \mathbb{R}^{m}$. Also, the transfer function associated with system (1) is defined as

$W(z)=D+C(z I-A)^{-1} B$,

where $z$ is a forward shift operator i.e. $z u_{k}=u_{k+1}$ and $z y_{k}=y_{k+1}$, and also represents a complex number.

Now we define for a fixed but arbitrary positive number $N>1$

$U_{k}=\left[\begin{array}{llll}u_{k}^{T} & u_{k+1}^{T} & \cdots & u_{k+N-1}^{T}\end{array}\right]^{T}$,

$Y_{k}=\left[\begin{array}{llll}y_{k}^{T} & y_{k+1}^{T} & \cdots & y_{k+N-1}^{T}\end{array}\right]^{T}$,

where $k=0, N, 2 N, \ldots$.

Then the blocked system is given by [3]

$x_{k+N}=A_{b} x_{k}+B_{b} U_{k}$,

$Y_{k}=C_{b} x_{k}+D_{b} U_{k}$,

where,

$A_{b}=A^{N}, \quad B_{b}=\left[\begin{array}{llll}A^{N-1} B & A^{N-2} B & \cdots & B\end{array}\right]$,

$C_{b}=\left[\begin{array}{llll}C^{T} & A^{T} C^{T} & \cdots & A^{(N-1)^{T}} C^{T}\end{array}\right]^{T}$,

$D_{b}=\left[\begin{array}{cccc}D & 0 & \cdots & 0 \\ C B & D & \cdots & 0 \\ \vdots & \vdots & \ddots & \vdots \\ C A^{N-2} B & C A^{N-3} B & \cdots & D\end{array}\right]$.

An operator $Z$ is defined such that $Z x_{k}=x_{k+N}, Z U_{k}=$ $U_{k+N}, Z Y_{k}=Y_{k+N}$. The symbol $Z$ is also used to denote a complex value. Then the transfer function of (4) is denoted by

$V(Z)=D_{b}+C_{b}\left(Z I-A_{b}\right)^{-1} B_{b}$

Furthermore, it is worthwhile remarking that the unblocked system (1) is a minimal realization of $W(z)$ if and only if the blocked system (4) is a minimal realization of $V(Z)$ [6].

\subsection{Unblocked systems and blocked systems-the transfer function}

In the previous subsection the state space representation for both unblocked and blocked systems was recalled $[9,18,10]$. The aim of this subsection is to recall a relation between $V(Z)$ and $W(z)$. The well-known result of $[6,9]$ is summarized as the theorem below.

Theorem 2.1. Consider the unblocked system (1) with transfer function $W(z)$ and the blocked system (4) with transfer function $V(Z)$. Then

$$
V(Z)=\left[\begin{array}{ccccc}
V_{1}(Z) & Z^{-1} V_{N}(Z) & Z^{-1} V_{N-1}(Z) & \cdots & Z^{-1} V_{2}(Z) \\
V_{2}(Z) & V_{1}(Z) & Z^{-1} V_{N}(Z) & \cdots & Z^{-1} V_{3}(Z) \\
V_{3}(Z) & V_{2}(Z) & V_{1}(Z) & \cdots & Z^{-1} V_{4}(Z) \\
\vdots & \vdots & \vdots & \ddots & \vdots \\
V_{N}(Z) & V_{N-1}(Z) & V_{N-2}(Z) & \cdots & V_{1}(Z)
\end{array}\right]
$$

and

$W(z)=V_{1}\left(z^{N}\right)+z^{-1} V_{2}\left(z^{N}\right)+\cdots+z^{-(N-1)} V_{N}\left(z^{N}\right)$,

where $V_{1}(Z)=D+C\left(Z I-A^{N}\right)^{-1} A^{N-1} B$ and $V_{l}(Z)=C A^{l-2} B+C(Z I-$ $\left.A^{N}\right)^{-1} A^{N+l-2} B, l=2, \ldots, N$. 
Another important result regarding the relation between $V(Z)$ and $W(z)$ is recorded in $[18,10]$. Assume that the transfer function of the unblocked system (1) is represented by a polynomial left coprime matrix fraction description (MFD) as

$W(z)=Q^{-1}(z) P(z)$,

where

$P(z)=P_{\mu}+P_{\mu-1} z+\cdots+P_{0} z^{\mu}$,

$Q(z)=Q_{\mu}+Q_{\mu-1} z+\cdots+Q_{0} z^{\mu}$.

In the above equation, $\mu$ is defined such that $P_{0}$ and $Q_{0}$ are not both zero. By coprimeness, $P_{\mu}$ and $Q_{\mu}$ are not both zero. Then it can be easily shown that associated with the blocked system there exists a transfer function with a polynomial left matrix fraction description as below

$Y_{k}=V(Z) U_{k}, \quad V(Z)=\mathcal{A}^{-1}(Z) B(Z)$,

where

$\mathcal{A}(Z)=\mathcal{A}_{0}+\mathcal{A}_{1} Z+\cdots+\mathcal{A}_{\alpha} Z^{\alpha}+\mathcal{A}_{\alpha+1} Z^{\alpha+1}$,

$\mathscr{B}(Z)=\mathscr{B}_{0}+\mathscr{B}_{1} Z+\cdots+\mathscr{B}_{\alpha} Z^{\alpha}+\mathscr{B}_{\alpha+1} Z^{\alpha+1}$,

where $\alpha$ is the greatest integer less than $\mu / N$ and $\mathcal{A}_{i}, \mathscr{B}_{i}, i \in$ $\{0,1, \ldots, \alpha+1\}$ are constant coefficient matrices of size $N(p \times$ $m$ ) obtained by a certain procedure from the coefficient matrices $P_{i}, Q_{i}, i \in\{0,1, \ldots, \mu\}$, respectively [10].

In the above, we related $V(Z)$ and $W(z)$. However, by using the above calculation relating the $\mathcal{B}_{i}$ to $P_{l}$, we are able to relate $\mathcal{B}(Z)$ and $P(z)$ as well. The following lemma is adapted from Lemma 2 in [10] and relates the $\mathcal{B}(Z)$ and $P(z)$.

Lemma 2.2. For a nonzero complex number $Z_{0}$, let $z_{i}, i=1$, $2, \ldots, N$, be $N$ distinct complex numbers such that $z_{i}^{N}=Z_{0}, i=$ $1,2, \ldots, N$

$\Upsilon=\left[\begin{array}{cccc}I_{m} & I_{m} & \cdots & I_{m} \\ z_{1} I_{m} & z_{2} I_{m} & \cdots & z_{N} I_{m} \\ \vdots & \ddots & \ddots & \vdots \\ z_{1}^{N-1} I_{m} & z_{2}^{N-1} I_{m} & \cdots & z_{N}^{N-1} I_{m}\end{array}\right]$,

$\Lambda=\left[\begin{array}{cccc}P\left(z_{1}\right) & P\left(z_{2}\right) & \cdots & P\left(z_{N}\right) \\ z_{1} P\left(z_{1}\right) & z_{2} P\left(z_{2}\right) & \cdots & z_{N} P\left(z_{N}\right) \\ \vdots & \ddots & \ddots & \vdots \\ z_{1}^{N-1} P\left(z_{1}\right) & z_{2}^{N-1} P\left(z_{2}\right) & \cdots & z_{N}^{N-1} P\left(z_{N}\right)\end{array}\right]$.

Then

$\mathscr{B}\left(Z_{0}\right) \Upsilon=\Lambda$.

Proof. The proof can be done in a similar way as the proof of Lemma 2 in [10].

The results obtained in this section help us to analyze the zero properties of the blocked system (4) in the following section.

\section{Zero properties of blocked systems}

In this section, the definitions for zeros of the systems (4) and (1) are first reviewed. Then, the zero properties of blocked systems are studied. Since the analysis of the zero properties for blocked systems is quite complicated, we consider three cases separately, that is, (1) finite nonzero system zeros; (2) system zeros at infinity; and (3) system zeros at zero. Finally, the last subsection covers results for the zero properties of the system (4) with a generic choice of matrices $A, B$, etc.

\subsection{Definition}

In order to study the zero properties of the system (4), we need to provide a precise definition for zeros. Here, we first recall the following definition for zeros of the unblocked system (1) from $[13,19]$ (p. 178).

Definition 3.1. The finite zeros of the transfer function $W(z)=$ $C(z I-A)^{-1} B+D$ with minimal realization $\{A, B, C, D\}$ are defined to be the finite values of $z$ for which the rank of the following system matrix falls below its normal rank:

$M(z)=\left[\begin{array}{cc}z I-A & -B \\ C & D\end{array}\right]$.

Further, $W(z)$ is said to have an infinite zero when $n+\operatorname{rank}(D)$ is less than the normal rank of $M(z)$, or equivalently the rank of $D$ is less than the normal rank of $W(z)$.

Similar to the above definition, we state the following definition for zeros of the blocked system (4).

Definition 3.2. The finite zeros of the transfer function $V(Z)=$ $C_{b}\left(Z I-A_{b}\right)^{-1} B_{b}+D_{b}$ with minimal realization $\left\{A_{b}, B_{b}, C_{b}, D_{b}\right\}$ are defined to be the finite values of $Z$ for which the rank of the following system matrix falls below its normal rank

$M_{b}(Z)=\left[\begin{array}{cc}Z I-A_{b} & -B_{b} \\ C_{b} & D_{b}\end{array}\right]$.

Further, $V(Z)$ is said to have an infinite zero when $n+\operatorname{rank}\left(D_{b}\right)$ is less than the normal rank of $M_{b}(Z)$, or equivalently the rank of $D_{b}$ is less than the normal rank of $V(Z)$.

\subsection{Blocked systems and unblocked systems-the normal rank}

As shown in the last subsection, the normal rank plays an important role in characterization of zeros. Thus, in this subsection an important result regarding the relation between the normal rank of $V(Z)$ and the normal rank of $W(z)$ is given. A restrictive version of the following result is initially stated as Theorem 3 in [10] only for linear time-invariant systems with $p \geq m$ and fullcolumn normal rank. Here we extend this results to linear timeinvariant systems with an arbitrary normal rank.

Theorem 3.3. Consider the unblocked transfer function $W(z)$ given by (2) and the blocked transfer function $V(Z)$ given (6). Then the following equality relates their normal rank:

normal rank $(V(Z))=N \times$ normal $\operatorname{rank}(W(z))$

Proof. To prove the conclusion of theorem, we modify the proof of Theorem 3 in [10]. First let $r$ and $S$ denote the normal rank of $W(z)$ and the normal rank of $V(Z)$, respectively.

There exists a complex number $Z_{0} \neq 0$ and $N$ distinct complex numbers $z_{i}, i=1,2, \ldots, N$ such that $\operatorname{det}\left(\mathcal{A}\left(Z_{0}\right)\right) \neq 0$, $\operatorname{rank}\left(\mathcal{B}\left(Z_{0}\right)\right)=S, z_{i}^{N}=Z_{0}, i=1,2, \ldots, N$ and $\operatorname{rank}\left(P\left(z_{i}\right)\right)=$ $r, i=1,2, \ldots, N$. Define $\Upsilon$ and $\Lambda$ as in (13), then it follows from Lemma 2.2 that $B\left(Z_{0}\right) \Upsilon=\Lambda$. Noting that $z_{i} \neq z_{l}$ for $i \neq l$, we see that $\Upsilon$ is a nonsingular matrix because it is a Kronecker product of a nonsingular VanderMonde matrix with the identity matrix. Furthermore, $\Lambda$ can be written as

$$
\begin{aligned}
\Lambda= & {\left[\begin{array}{cccc}
I_{p} & I_{p} & \cdots & I_{p} \\
z_{1} I_{p} & z_{2} I_{p} & \ldots & z_{N} I_{p} \\
\vdots & \ddots & \ddots & \vdots \\
z_{1}^{N-1} I_{p} & z_{2}^{N-1} I_{p} & \cdots & z_{N}^{N-1} I_{p}
\end{array}\right] } \\
& \times\left[\begin{array}{cccc}
P\left(z_{1}\right) & 0 & \cdots & 0 \\
0 & P\left(z_{2}\right) & \cdots & 0 \\
\vdots & \ddots & \ddots & \vdots \\
0 & 0 & \cdots & P\left(z_{N}\right)
\end{array}\right] \\
= & \tilde{\Upsilon} \operatorname{diag}\left\{P\left(z_{1}\right), P\left(z_{2}\right), \ldots, P\left(z_{N}\right)\right\} .
\end{aligned}
$$


Observe that $\tilde{\Upsilon}$ is a nonsingular matrix. Hence, $\Lambda$ has rank $N r$ which implies that $\operatorname{rank}\left(\mathscr{B}\left(Z_{0}\right)\right)=\operatorname{rank}(\Lambda)=N r$. This together with the fact that $\operatorname{det}\left(\mathcal{A}\left(Z_{0}\right)\right) \neq 0$ implies that $\operatorname{rank}\left(V\left(Z_{0}\right)\right)=$ $\operatorname{rank}\left(\mathcal{A}^{-1}\left(Z_{0}\right) \mathscr{B}\left(Z_{0}\right)\right)=N r$. Since the rank of $V(Z)$ for a particular choice of $Z$ is equal to $N r$; thus, the normal rank of $V(Z) \geq N r$ i.e. $S \geq N r$.

Conversely, there exists a complex number $Z_{0} \neq 0$ such that $\operatorname{det}\left(\mathcal{A}\left(Z_{0}\right)\right) \neq 0$ and $\mathcal{B}\left(Z_{0}\right)$ has $\operatorname{rank} S$. Now let $z_{i}, i=1,2, \ldots, N$ be complex numbers such that $z_{i}^{N}=Z_{0}, i=1,2, \ldots, N$ and $\operatorname{rank}\left(P\left(z_{i}\right)\right)=\operatorname{rank}\left(P\left(z_{l}\right)\right), z_{i} \neq z_{l}$. Define $\Upsilon$ and $\Lambda$ as in (13), then it follows from Lemma 2.2 that $B\left(Z_{0}\right) \Upsilon=\Lambda$. Noting that $z_{i} \neq z_{l}$ for $i \neq l$ so, $\Upsilon$ is nonsingular. Hence, $\Lambda$ has rank $S$. Hence, it follows from the definition of $\Lambda$ that all $P\left(z_{i}\right), i \in\{1,2, \ldots, N\}$ have the same rank equal to $S / N$, (which must therefore be an integer). Since for particular $z_{i}$, we have $P\left(z_{i}\right)$ and thus $W\left(z_{i}\right)$ of rank $S / N$ there holds normal rank $W(z) \geq S / N$ or $N r \geq S$.

Now, by using the both inequalities i.e. $N r \geq S$ and $S \geq N r$, we can conclude that $S=N r$.

The above theorem relates the normal rank of associated unblocked and blocked transfer functions. We can also relate the normal rank of associated system matrices to the respective transfer functions and to each other.

Lemma 3.4. Consider the unblocked transfer function $W(z)$ given by (2) and its corresponding system matrix denoted by $M(z)$. Then the following equality holds:

normal $\operatorname{rank}(M(z))=n+$ normal $\operatorname{rank}(W(z))$

Proof.

$$
\begin{aligned}
M(z) & =\left[\begin{array}{cc}
z I-A & -B \\
C & D
\end{array}\right] \\
& =\left[\begin{array}{cc}
I & 0 \\
C(z I-A)^{-1} & I
\end{array}\right]\left[\begin{array}{cc}
z I-A & -B \\
0 & W(z)
\end{array}\right] .
\end{aligned}
$$

Observe that the normal $\operatorname{rank}(M(z))=\operatorname{normal} \operatorname{rank}(z I-A)+$ normal $\operatorname{rank}(W(z))=n+$ normal $\operatorname{rank}(W(z))$.

Corollary 3.5. The normal rank of $M(z)$ is $n+r$ if and only if the normal rank of $M_{b}(Z)$ is $n+N r$.

Proof. The proof is immediate using the results of Lemma 3.4 and Theorem 3.3.

\subsection{Blocked systems and unblocked systems-zeros}

In the last subsection the relation between the normal rank of $V(Z)$ and the normal rank of $W(z)$ was explored. In this subsection, the relation between zeros of blocked systems and those of their corresponding unblocked systems is investigated. As stated earlier, due to the complexity of analysis, we consider three cases separately, that is, (1) finite nonzero system zeros; (2) system zeros at infinity; and (3) system zeros at zero.

A sufficient condition under which the blocked system (4) has a finite nonzero zero has been provided in [6]. Later, in [10,21] a necessary and sufficient condition has been provided where a tall blocked system with full-column normal rank has a finite nonzero zero. Here, we provide the following necessary and sufficient condition for the blocked system (4) to have a finite nonzero zero without imposing any condition either on the structure of the blocked system or on the normal rank of its associated transfer function matrix.

Theorem 3.6. Consider the unblocked system (1) with transfer function $W(z)$ given by (2) and the blocked system (4) with transfer function $V(Z)$ given by (6). Suppose that the quadruple $\{A, B, C, D\}$ is minimal. Define $\omega \triangleq \exp \left(\frac{2 \pi j}{N}\right)$. It follows that
1. If $W(z)$ has a finite zero at $z_{0} \neq 0$ then $V(Z)$ has a finite zero at $Z=Z_{0}=z_{0}^{N} \neq 0$.

2. If $V(Z)$ has a finite zero at $Z=Z_{0} \neq 0$ then, for any $z_{0}$ satisfying $z_{0}^{N}=Z_{0}, W(z)$ has a finite zero at one or more of $z=z_{0} \neq 0$ or $z=\omega z_{0} \neq 0 \ldots z=\omega^{N-1} z_{0} \neq 0$.

Proof. We prove the first part of the theorem. Let $r$ denote the normal rank of $W(z)$. Assume that the unblocked system has a zero at $z_{0}$ which implies that $\operatorname{rank}\left(P\left(z_{0}\right)\right)<r$. Now, let $z_{i}=$ $\omega^{i} z_{0}, i=1,2, \ldots, N$, where $\omega=\exp \left(\frac{2 \pi j}{N}\right)$, be $N$ distinct complex numbers and $z_{i}^{N}=z_{0}^{N}=Z_{0}, i=1,2, \ldots, N$. One can define $\Upsilon$ and $\Lambda$ as in (13); then using Lemma 2.2 it is immediate that $\mathscr{B}\left(Z_{0}\right) \Upsilon=\Lambda$. Moreover, with the help of Eq. (17), we can obtain $\mathscr{B}\left(Z_{0}\right) \Upsilon=\widetilde{\Upsilon} \operatorname{diag}\left\{P\left(z_{1}\right), P\left(z_{2}\right), \ldots, P\left(z_{N}\right)\right\}$. Since $z_{i}$ are chosen to be distinct $\Upsilon$ and $\widetilde{\Upsilon}$ are nonsingular matrices and $\operatorname{rank}\left(\mathscr{B}\left(Z_{0}\right)\right)=$ $\operatorname{rank}\left(\operatorname{diag}\left\{P\left(z_{1}\right), P\left(z_{2}\right), \ldots, P\left(z_{N}\right)\right\}\right)$. Since $\operatorname{rank}\left(P\left(z_{i}\right)\right) \leq r, i=$ $1,2, \ldots, N-1$, the assumption that $\operatorname{rank}\left(P\left(z_{0}\right)\right)<r$ implies that $\operatorname{rank}\left(\mathscr{B}\left(Z_{0}\right)\right)<N r$ so, $\operatorname{rank}\left(V\left(Z_{0}\right)\right)<N r$. Furthermore, from Theorem 3.3, it is known that normal $\operatorname{rank}(V(Z))=N r$; hence, the blocked system (4) has a finite zero at $Z_{0}=z_{0}^{N}$.

We now turn to the part two. Suppose that $Z_{0}$ is a zero for the system matrix of (4). Also, note that there exist $N$ distinct complex numbers $z_{i}, i \in\{1,2, \ldots, N\}$ such that $z_{i}^{N}=Z_{0}, i \in\{1,2, \ldots, N\}$. Hence, according to the result of Lemma $2.2, \Upsilon$ is a nonsingular matrix and $\operatorname{rank}\left(\mathscr{B}\left(Z_{0}\right)\right)=\operatorname{rank}(\Lambda)$. Since $\operatorname{rank}(\Lambda)$ is less than normal rank, one or more of $P\left(z_{i}\right), i \in\{1,2, \ldots, N\}$ should have less than the normal rank. The latter implies that the system (1) has a finite zero at one or more of $z=z_{0} \neq 0$ or $z=\omega z_{0} \neq$ $0 \ldots z=\omega^{N-1} z_{0} \neq 0$.

Remark 3.7. It is worthwhile mentioning that the factorization recalled in Remark 1 of [10], can also be used to prove the statement of the above theorem.

The above theorem treats the zero properties of the blocked system for choice of finite nonzero zeros; it is natural to ask what happens to zeros at infinity, and the following theorem deals with this case.

Theorem 3.8. Consider the unblocked system (1) with transfer function $W(z)$ given by (2) and the blocked system (4) with transfer function $V(Z)$ given by (6). Suppose that the quadruple $\{A, B, C, D\}$ is minimal. Then $W(z)$ has a zero at $z=\infty$ if and only if $V(Z)$ has a zero at $Z=\infty$.

Proof. Sufficiency. We first relate the rank of $D_{b}$ and the rank of $D$ (here the case where $N=2$ is discussed and generalization to an arbitrary $N$ is straightforward). Suppose that $D$ has $\operatorname{rank} q$, then there exist invertible matrices $\bar{T}$ and $\bar{S}$ such that $\bar{S} D \bar{T}=\left[\begin{array}{cc}I_{q} & 0 \\ 0 & 0\end{array}\right]$. Then one can write

$\left[\begin{array}{ll}\bar{S} & 0 \\ 0 & \bar{S}\end{array}\right]\left[\begin{array}{cc}D & 0 \\ C B & D\end{array}\right]\left[\begin{array}{cc}\bar{T} & 0 \\ 0 & \bar{T}\end{array}\right]=\left[\begin{array}{cc}\bar{S} D \bar{T} & 0 \\ \bar{S} C B \bar{T} & \bar{S} D \bar{T}\end{array}\right]$.

It now becomes immediate that $\operatorname{rank}\left(D_{b}\right) \geq 2 q$ i.e. $\operatorname{rank}\left(D_{b}\right) \geq$ $2 \operatorname{rank}(D)$, and in a general case with an arbitrary $N$ we have $\operatorname{rank}\left(D_{b}\right) \geq N \operatorname{rank}(D)$.

Now suppose that $V(Z)$ has a zero at infinity and let $r$ denote the normal rank of $W(z)$, then according to the Definition 3.2, the matrix $\operatorname{rank}\left(D_{b}\right)<N r$. Now, using the result of Theorem 3.3 one can write $\operatorname{rank}\left(D_{b}\right)<N \times$ normal $\operatorname{rank}(W(z))$. Then it becomes immediate that $\operatorname{rank}(D)<r$ which implies that $W(z)$ has a zero at infinity.

Necessity. The proof of necessity part is immediate by using the structure of $D_{b}$. 
Finally in the remainder of this subsection, we examine the zero properties of blocked systems for zeros at the origin. In order to deal with this case we first need to review the following result from [20], obtained by specializing Lemma 1 of [20] to the case where the unblocked system is time-invariant.

Lemma 3.9 ([20]). Let $\widetilde{A}_{b}=I_{N} \otimes A, \widetilde{B}_{b}=I_{N} \otimes B, \widetilde{C}_{b}=I_{N} \otimes C$ and $\widetilde{D}_{b}=I_{N} \otimes D$. Furthermore, define $E_{Z} \triangleq\left[\begin{array}{cccc}0 & 1 & & 0 \\ 0 & & \ddots & \\ \vdots & & \ddots & 1 \\ Z & 0 & & 0\end{array}\right], E_{Z} \in \mathbb{C}^{n \times n}$ and $\widetilde{E}_{Z}=E_{Z} \otimes I_{N}$ and $\otimes$ denotes the Kronecker product. Then there exist invertible matrices $T_{l}$ and $T_{r}$ and matrices $X$ and $Y$ such that for all $Z \in \mathbb{C}$

$$
\begin{aligned}
& {\left[\begin{array}{ccc}
I_{n(N-1)} & 0 & 0 \\
0 & Z I-A_{b} & -B_{b} \\
0 & C_{b} & D_{b}
\end{array}\right]} \\
& =\left[\begin{array}{cc}
T_{l} & 0 \\
X & I
\end{array}\right]\left[\begin{array}{cc}
\widetilde{E}_{Z}-\widetilde{A}_{b} & -\widetilde{B}_{b} \\
\widetilde{C}_{b} & \widetilde{D}_{b}
\end{array}\right]\left[\begin{array}{cc}
T_{r} & Y \\
0 & I
\end{array}\right] .
\end{aligned}
$$

Theorem 3.10. Consider the unblocked system (1) with transfer function $W(z)$ given by (2) and the blocked system (4) with transfer function $V(Z)$ given by (6). Suppose that the quadruple $\{A, B, C, D\}$ is minimal. Then $W(z)$ has a zero at $z=0$ if and only if $V(Z)$ has a zero at $Z=0$.

Proof. Sufficiency. Let $W(z)$ have normal rank $r$ so, $V(Z)$ has normal rank $N r$ and $M_{b}(Z)$ has normal rank $n+N r$. Now suppose that $V(Z)$ has a zero at $Z=0$, so that $M_{b}(Z)$ has rank less than its normal rank at $Z=0$. Then rank $\left[\begin{array}{ccc}I_{n(N-1)} & 0 & 0 \\ 0 & -A_{b} & -B_{b} \\ 0 & C_{b} & D_{b}\end{array}\right]<N(n+r)$. Now by using the conclusion of the Lemma 3.9, we can write

$$
\begin{aligned}
& {\left[\begin{array}{ccc}
I_{n(N-1)} & 0 & 0 \\
0 & -A_{b} & -B_{b} \\
0 & C_{b} & D_{b}
\end{array}\right]} \\
& =\left[\begin{array}{ll}
T_{l} & 0 \\
X & I
\end{array}\right]\left[\begin{array}{cc}
E_{0}-\widetilde{A}_{b} & -\widetilde{B}_{b} \\
\widetilde{C}_{b} & \widetilde{D}_{b}
\end{array}\right]\left[\begin{array}{cc}
T_{r} & Y \\
0 & I
\end{array}\right]
\end{aligned}
$$

and so rank $\left[\begin{array}{cc}E_{0}-\widetilde{A}_{b} & -\widetilde{B}_{b} \\ \widetilde{C}_{b} & \widetilde{D}_{b}\end{array}\right]<N(n+r)$.

On the other hand, $\operatorname{rank}\left[\begin{array}{cc}E_{0}-\widetilde{A}_{b} & -\widetilde{B}_{b} \\ \widetilde{C}_{b} & \widetilde{D}_{b}\end{array}\right] \geq \operatorname{Nrank}\left[\begin{array}{cc}-A & -B \\ C & D\end{array}\right]$. (This follows from the fact that with row and column reordering the matrix on the left can be made upper triangular with diagonal blocks all of the form $\left.\left[\begin{array}{cc}-A & -B \\ C & D\end{array}\right]\right)$. The last two inequalities imply that rank $\left[\begin{array}{cc}-A & -B \\ C & D\end{array}\right]<n+$ rand so the unblocked system has a zero at $z=0$.

Necessity. Suppose $W(z)$ has a zero at $z=0$. Note that the associated system is finite dimensional and therefore $A$ has only a finite number of eigenvalues. Thus there exists $\rho>0$ such that $A-z I$ is invertible for all real numbers $z$ with $0<z<\rho$. Let $\epsilon$ be any such number. (We shall further restrict $\epsilon$ subsequently). Let $A_{\epsilon} \triangleq A-\epsilon I$; hence, $A_{\epsilon}$ is nonsingular. Clearly, $z=0$ is a point where there is rank loss of the system matrix associated with the quadruple $\{A, B, C, D\}$ if and only if $z=\epsilon$ is a point where there is rank loss of the system matrix associated with the quadruple $\left\{A_{\epsilon}, B, C, D\right\}$. Using the result of the Theorem 3.6, we can conclude that $\epsilon^{N}$ is a point where there is rank loss of the system matrix associated with a quadruple $\left\{\bar{A}_{b_{\epsilon}}, \bar{B}_{b_{\epsilon}}, \bar{C}_{b_{\epsilon}}, \bar{D}_{b_{\epsilon}}\right\}$ (where the quadruple $\left\{\bar{A}_{b_{\epsilon}}, \bar{B}_{b_{\epsilon}}, \bar{C}_{b_{\epsilon}}, \bar{D}_{b_{\epsilon}}\right\}$ characterizes the blocked system associated with the quadruple $\left.\left\{A_{\epsilon}, B, C, D\right\}\right)$. By hypothesis, the following inequality holds

$$
\begin{aligned}
& \operatorname{rank}\left[\begin{array}{cc}
\epsilon^{N} I-\bar{A}_{b_{\epsilon}} & \bar{B}_{b_{\epsilon}} \\
\bar{C}_{b_{\epsilon}} & \bar{D}_{b_{\epsilon}}
\end{array}\right] \\
& \quad<\text { normal rank }\left[\begin{array}{cc}
Z I-\bar{A}_{b_{\epsilon}} & \bar{B}_{b_{\epsilon}} \\
\bar{C}_{b_{\epsilon}} & \bar{D}_{b_{\epsilon}}
\end{array}\right] .
\end{aligned}
$$

With the help of Eq. (18), one can write

$$
\begin{aligned}
& n(N-1)+\text { normal rank }\left[\begin{array}{cc}
Z I-A_{b} & -B_{b} \\
C_{b} & D_{b}
\end{array}\right] \\
& \quad=\text { normal rank }\left[\begin{array}{cc}
E_{Z}-\widetilde{A}_{b} & -\widetilde{B}_{b} \\
\widetilde{C}_{b} & \widetilde{D}_{b}
\end{array}\right]
\end{aligned}
$$

and

$$
\begin{aligned}
& n(N-1)+\text { normal rank }\left[\begin{array}{cc}
Z I-\bar{A}_{b_{\epsilon}} & \bar{B}_{b_{\epsilon}} \\
\bar{C}_{b_{\epsilon}} & \bar{D}_{b_{\epsilon}}
\end{array}\right] \\
& \quad=\text { normal rank }\left[\begin{array}{cc}
E_{Z}-\widetilde{A}_{b_{\epsilon}} & -\widetilde{B}_{b} \\
\widetilde{C}_{b} & \widetilde{D}_{b}
\end{array}\right]
\end{aligned}
$$

where $\widetilde{A}_{b_{\epsilon}}=I_{N} \otimes A_{\epsilon}$. We now show that

$$
\begin{gathered}
\text { normal rank }\left[\begin{array}{cc}
Z I-\bar{A}_{b_{\epsilon}} & \bar{B}_{b_{\epsilon}} \\
\bar{C}_{b_{\epsilon}} & \bar{D}_{b_{\epsilon}}
\end{array}\right] \\
\quad \text { = normal rank }\left[\begin{array}{cc}
Z I-A_{b} & -B_{b} \\
C_{b} & D_{b}
\end{array}\right] .
\end{gathered}
$$

Suppose that $W(z)=D+C(z I-A)^{-1} B$ and normal $\operatorname{rank}(W(z))$ $=r, r \leq \min \{m, p\}$, then $W_{\epsilon}(z) \triangleq D+C\left(z I-A_{\epsilon}\right)^{-1} B=$ $D+C((z+\epsilon) I-A)^{-1} B$ has the same normal rank i.e. $r$. By using the result of Theorem 3.3, we have normal $\operatorname{rank}(V(Z))=N r$ and normal $\operatorname{rank}\left(V_{\epsilon}(Z)\right)=N r$ (where $V_{\epsilon}(Z)$ is the transfer function associated the quadruple $\left.\left\{\bar{A}_{b_{\epsilon}}, \bar{B}_{b_{\epsilon}}, \bar{C}_{b_{\epsilon}}, \bar{D}_{b_{\epsilon}}\right\}\right)$. Thus, with the help of Lemma 3.4, the equality (21) becomes immediate.

At this stage of proof we illustrate that

$\operatorname{rank}\left(M_{b}(0)\right) \leq \operatorname{rank}\left(M_{b_{\epsilon}}\left(\epsilon^{N}\right)\right)$,

where $M_{b_{\epsilon}}\left(\epsilon^{N}\right)=\left[\begin{array}{cc}\epsilon^{N} I-\bar{A}_{b_{\epsilon}} & \bar{B}_{b_{\epsilon}} \\ \bar{C}_{b_{\epsilon}} & \bar{D}_{b_{\epsilon}}\end{array}\right]$.

Suppose that rank $\left[\begin{array}{cc}-A^{N} & B_{b} \\ C_{b} & D_{b}\end{array}\right]=q$; then there exists a $q \times q$ submatrix of $M_{b}(0)$ such that its determinant is nonzero. Recall that the determinant function is a continuous function of the entries of the associated matrix and the quadruple $\left\{\bar{A}_{b_{\epsilon}}, \bar{B}_{b_{\epsilon}}, \bar{C}_{b_{\epsilon}}, \bar{D}_{b_{\epsilon}}\right\}$ can be obtained from $\left\{A_{b}, B_{b}, C_{b}, D_{b}\right\}$ with a perturbation $\epsilon$ of the underlying unblocked matrix $A$. Therefore, since $\rho$ can be chosen arbitrarily small while positive, we may assume that for any positive $\epsilon$ with $\epsilon<\rho$, there exists a $q \times q$ submatrix of $M_{b_{\epsilon}}\left(\epsilon^{N}\right)$ which has nonzero determinant, so that rank $\left[\begin{array}{ccc}\epsilon^{N} I-\bar{A}_{b_{\epsilon}} & \bar{B}_{b_{\epsilon}} \\ \bar{c}_{b_{\epsilon}} & \bar{D}_{b_{\epsilon}}\end{array}\right] \geq q$.

Finally, by combining (20)-(22), we obtain

$\operatorname{rank}\left[\begin{array}{cc}-A^{N} & B_{b} \\ C_{b} & D_{b}\end{array}\right]<$ normal rank $\left[\begin{array}{cc}Z I-A_{b} & -B_{b} \\ C_{b} & D_{b}\end{array}\right]$.

Equivalently, $Z=0$ is a zero for $V(Z)$.

In this subsection we have studied the zero properties of the blocked system for the whole complex plane including infinity. One can naturally ask what are the zero properties of the blocked system for a generic choice of parameter matrices $A, B$, etc. It is important to recall that the zero properties of the unblocked system (1) for a generic choice of parameter matrices have been studied in the literature (see e.g. $[13,15,12]$ ). However, since the 
parameter matrices of the blocked system (4) are structured and their entries cannot independently assume generic values, the study of zero properties in this case is not trivial.

\subsection{Zeros properties of blocked systems under a generic setting}

The following theorem investigates the zero properties of the system (4) for a generic choice of system matrices $A, B, C, D$. It extends the earlier result of [21], which only considered tall blocked systems. Ref. [21] states that tall blocked systems have no zeros when matrices $A, B, C, D$ assume generic values. The following theorem generalizes the result in [21] by specifying that nonsquare blocked systems have no zeros when matrices $A, B, C, D$ accept generic values and square blocked systems generically only have finite zeros and these finite zeros have geometric multiplicity one.

Theorem 3.11. Consider the system (1) defined by the quadruple $\{A, B, C, D\}$, in which the individual matrices are generic and $m, n, p$ are nonzero. Then

1. If $p>m$, the system matrix of the blocked system has full column rank for all $Z$.

2. If $p<m$, the system matrix of the blocked system has full row rank for all $Z$.

3. If $p=m$, then the system matrix of the blocked system must have finite zeros with one-dimensional kernel.

Proof. Suppose first that $p>m$. Then it can be readily shown that the system matrix of tall unblocked systems generically have full-column normal rank. Furthermore, [15] showed that for tall unblocked systems are generically zero-free. If the blocked system had its system matrix with less than full column rank for a finite $Z_{0} \neq 0$, then according to Theorem 3.6, there would be necessarily a nonzero nullvector of the system matrix of the unblocked system for $z_{0} \neq 0$ equal to some $N$-th root of $Z_{0}$, which would be a contradiction. If the blocked system had a zero at $Z_{0}=\infty$, then based on Theorem 3.8 the $D$ matrix of the unblocked system would be less than full column rank which would be a contradiction. Analogously, using the argument in Theorem 3.10, one can easily conclude that blocked system has full column rank system matrix at $Z_{0}=0$. The case $p<m$ can be done similarly. (One can appeal to the fact, demonstrated in [6] see p. 180, that if $V(Z)$ is the blocked transfer function associated with $W(z)$, then $P_{1} V^{T}(Z) P_{2}$ for certain permutation matrices $P_{1}, P_{2}$ is the blocked transfer function associated with $\left.W^{T}(z)\right)$.

Now we consider the case $p=m$; since $D$ is generic, it has full column rank. Hence, based on the conclusion of Theorem 3.8, both the unblocked system and the blocked system do not have zeros at infinity. In the second part of this proof we use the conclusion of Theorem 3.6. Furthermore, one should note that since matrices $A, B, C$ and $D$ assume generic values it can be easily understood that the quadruple $\left\{A_{b}, B_{b}, C_{b}, D_{b}\right\}$ is a minimal realization. Now, based on the fact that $D_{b}$ is nonsingular, one can conclude that the zeros of the blocked system are the eigenvalues of $A_{b}-B_{b} D_{b}^{-1} C_{b}$. If the eigenvalues of $A_{b}-B_{b} D_{b}^{-1} C_{b}$ are distinct, then the associated eigenspace for each eigenvalue is one-dimensional; it is equivalent to saying that the associated kernel of $M_{b}(Z)$ evaluated at the eigenvalue has dimension one. One should note that the unblocked system has distinct zeros due to the genericity assumption. Furthermore, zeros of the unblocked system generically have distinct magnitudes except for complex conjugate pairs. It is obvious that those zeros of the unblocked system with distinct magnitudes produce distinct blocked zeros. Now, we focus on zeros of the unblocked system with the same magnitudes, i.e. complex conjugate pairs. The only case where the generic unblocked system has distinct zeros but its corresponding blocked system has non-distinct zeros happens when the $\mathrm{N}$-th power of the complex conjugate zeros of the unblocked system coincide. We now show by contradiction that this is generically impossible. In order to illustrate a contradiction, suppose that the unblocked system has a complex conjugate pair, say $z_{01}$ and $z_{01}^{*}$. If they produce an identical zero for the blocked system, their $N$-th powers must be the same. The latter condition implies that the angle between $z_{01}$ and $z_{01}^{*}$ has to be exactly $\frac{2 \pi h}{N}$, where $h$ is an integer, which contradicts the genericity assumption for the unblocked system. Hence, the zeros of the blocked system generically have distinct values and consequently the corresponding kernels of system matrix evaluated at the zeros are one-dimensional.

\section{Conclusions}

The zero properties of the blocked system obtained from blocking of linear discrete time-invariant systems were studied in this paper. In particular it was shown that the blocked system is zerofree if and only if the related unblocked system is zero-free. In addition, the system matrix of the blocked system was investigated under the genericity assumption. It was demonstrated that blocked system generically has no zeros when it is either fat or tall. However, when the blocked system is square, it generically has a finite zero and the kernel associated with that zero is of dimension one.

\section{Acknowledgments}

The authors would like to thank Professor Manfred Deistler from the Department of Mathematical Methods in Economics, Technical University of Vienna for his valuable comments. Furthermore, support by the ARC Discovery Project Grant DP1092571, the FWF (Austrian Science Fund) under contracts P17378 and P20833/N18, DFG HE 1858/12-2 under the SPP 1305 program and the Oesterreichische Forschungsgemeinschaft is gratefully acknowledged.

\section{References}

[1] T. Chen, B.A. Francis, Optimal Sampled-Data Control Systems, Springer-Verlag New York, Inc., Secaucus, NJ, USA, 1995.

[2] P.P. Vaidyanathan, Multirate Systems and Filter Banks, Prentice-Hall, Inc. Upper Saddle River, NJ, USA, 1993.

[3] P. Bolzern, P. Colaneri, R. Scattolini, Zeros of discrete-time linear periodic systems, IEEE Transactions on Automatic Control 31 (11) (1986) 1057-1058.

[4] O.M. Grasselli, S. Longhi, Zeros and poles of linear periodic multivariable discrete-time systems, Circuits, Systems, and Signal Processing 7 (1988) 361-380.

[5] S. Bittanti, Deterministic and Stochastic Linear Periodic Systems, in: Lecture Notes in Control and Information Sciences, vol. 86, Springer, Berlin, Heidelberg, 1986.

[6] S. Bittanti, P. Colaneri, Periodic Systems Filtering and Control, in: Communications and Control Engineering, Springer-Verlag, 2009.

[7] O.M. Grasselli, S. Longhi, The geometric approach for linear periodic discretetime systems, Linear Algebra and its Applications 158 (1991) 27-60.

[8] P. Colaneri, S. Longhi, The realization problem for linear periodic systems, Automatica 31 (5) (1995) 775-779.

[9] P. Khargonekar, K. Poolla, A. Tannenbaum, Robust control of linear timeinvariant plants using periodic compensation, IEEE Transactions on Automatic Control 30 (11) (1985) 1088-1096.

[10] W. Chen, B.D.O. Anderson, M. Deistler, A. Filler, Properties of blocked linear systems, Automatica 48 (10) (2012) 2520-2525.

[11] W.M. Wonham, Linear Multivariable Control: A Geometric Approach, Springer-Verlag, New York, 1979.

[12] N. Karcanias, B. Kouvaritakis, The output zeroing problem and its relationship to the invariant zero structure: a matrix pencil approach, International Journal of Control 30 (3) (1979) 395-415.

[13] T. Kailath, Linear Systems, Prentice-Hall, New Jersey, 1980.

[14] N. Karcanias, D. Vafiadis, Canonical forms for state-space descriptions, Control Systems, Robotics and Automation 5 (2002) 361-380.

[15] A. Filler, Generalized dynamic factor models structure theory and estimation for single frequency and mixed frequency data, Ph.D. Thesis, Vienna University of Technology, 2010.

[16] D. Christou, N. Karcanias, M. Mitrouli, The ERES method for computing the approximate GCD of several polynomials, Applied Numerical Mathematics 60 (1-2) (2010) 94-114. 
[17] M. Mitrouli, N. Karcanias, Computation of the GCD of polynomials using Gaussian transformations and shifting, International Journal of Control 58 (1) (1993) 211-228.

[18] C. Burrus, Block realization of digital filters, IEEE Transactions on Audio and Electroacoustics 20 (4) (1972) 230-235.

[19] J.P. Hespanha, Linear Systems Theory, Princton University Press, 2009.
[20] A. Varga, P. Van Dooren, Computing the zeros of periodic descriptor systems, Systems and Control Letters 50 (5) (2003) 371-381.

[21] M. Zamani, W. Chen, B.D.O. Anderson, M. Deistler, A. Filler, On the zeros of blocked linear systems with single and mixed frequency data, in: Proceedings of the 50th IEEE Conference on Decision and Control and European Control Conference, CDC-ECC, 2011, pp. 4312-4317. 